Paru dans la Revue d'épidémiologie et de santé publique, fin 2009

Orientation sexuelle et santé mentale: une revue de la littérature

Sexual orientation and mental health: a review

B. Lhomond ${ }^{1}$, M.J. Saurel-Cubizolles ${ }^{2}$

1. Laboratoire Triangle, CNRS, ENS LSH, Université de Lyon

2. INSERM Unité 953-IFR69, Université Pierre et Marie Curie

Brigitte Lhomond, Triangle, ENS-LSH, 15 parvis René Descartes, 69342 Lyon cedex 07 Tel. : 0478381132

brigitte.lhomond@ens-lsh.fr

Titre courant : Orientation sexuelle et santé mentale 


\begin{abstract}
Objectives.- The aim of this paper is to review available knowledge on sexual orientation and mental health, especially for women.

Methods.- Papers published in English or French, between 1997 and 2007, were selected in PubMed using the following keywords: "homosexuality / sexual orientation and mental health/ depression/ suicide". To be retained papers had to contain findings from quantitative surveys comparing homosexual and heterosexual adults. In all, this review analyses 22 papers including two that are based on the same survey.

Results.- This review found a general pattern of poorer mental health for homosexuals and even more so for bisexuals compared to heterosexuals. Results are especially consistent regarding elevated risk of suicide attempts.
\end{abstract}

\title{
Résumé
}

Objectifs.- Le but de cet article est de faire le point des connaissances disponibles sur l'orientation sexuelle et la santé mentale, en particulier pour les femmes.

Méthode.- Les articles publiés en langue anglaise ou française, de 1997 à 2007, ont été sélectionnés dans Pubmed à l'aide des mots-clés suivants : «homosexuality / sexual orientation and mental health/ depression/ suicide». Pour être retenus, les articles devaient présenter des résultats d'enquêtes quantitatives, comparant la situation de personnes adultes « homosexuelles » à celle de personnes « hétérosexuelles ». Au total, cette revue analyse 22 articles, dont deux portent sur la même base de données.

Résultats.- Cette revue montre un tableau cohérent d'une moins bonne santé mentale pour les personnes homosexuelles et surtout bisexuelles. Les études sont particulièrement convergentes à propos du risque plus élevé de tentatives de suicide.

Keywords: Sexual orientation. Sexual behaviours. Mental health. Suicide.

Mots-clés: Orientation sexuelle. Comportements sexuels. Santé mentale. Suicide. 


\section{Orientation sexuelle et santé mentale: une revue de la littérature}

\section{Introduction}

Depuis une vingtaine d'années les études sur la santé des homosexuel-les se sont multipliées, essentiellement dans les pays anglo-saxons, en particulier aux Etats Unis. L'épidémie à VIH a été le moteur de nombre d'entre elles, ce qui implique que la grande majorité concerne les hommes et les infections sexuellement transmissibles, comme le montre la recension d'Ulrike Boehmer [1]. Un autre courant de recherche, plus ancien, centré sur l'homosexualité comme problème, a connu de formidables transformations. Le présupposé de la majorité de ces travaux, jusqu'aux années cinquante, était que l'homosexualité en tant que telle est une pathologie mentale, dans la suite de certaines théorisations de la psychiatrie depuis le milieu du $19^{\text {ème }}$ siècle. Dès cette période, le débat est vif entre les tenants de cette vision pathologique, par exemple Krafft-Ebing [2], et les défenseurs d'un point de vue que l'on pourrait qualifier aujourd'hui d'essentialiste, où l'homosexualité est vue comme une donnée de nature, par exemple Hirschfeld [3]. Des institutions comme l'American Psychiatric Association ou l'Organisation Mondiale de la Santé ont, jusqu'en 1974 pour la première et 1993 pour la seconde, considéré l'homosexualité comme une maladie mentale. Les travaux pionniers d'Evelyne Hooker sur les hommes homosexuels dans les années cinquante [4] et de Saghir et al. [5] sur les femmes aux Etats-Unis ont permis de remettre en question ce qui était alors largement admis, comme en témoigne par exemple l'autobiographie de Martin Duberman [6], en montrant que les homosexuel-les ne présentaient pas en soi de différences de santé psychique par rapport aux hétérosexuel-les. De plus, les enquêtes sur les comportements sexuels depuis Kinsey [7,8] ont modifié la perception, tant des scientifiques 
que du grand public, de la fréquence des comportements homosexuels et mis en évidence le continuum existant entre différentes expressions de la sexualité $[9,10]$.

Les mouvements homosexuels militants, du Wissenschaftlich Humanitärian Komittee (Comité humanitaire et scientifique) en Allemagne au début du $20^{\text {ème }}$ siècle [11] au Front Homosexuel d'Action Révolutionnaire en France dans les années 1970 [12], ont lutté pour la reconnaissance de l'homosexualité, sa décriminalisation et sa dé-psychiatrisation.

L'homosexualité est souvent considérée, dans les travaux scientifiques ainsi que dans les mouvements militants, comme l'homosexualité des hommes. L'inégalité sociale entre les hommes et les femmes s'exerce aussi dans l'homosexualité. La contrainte à l'hétérosexualité qui pèse plus fortement sur les femmes, la stigmatisation légale et sociale qui s'exerce plus souvent sur les hommes sont des exemples du traitement différentiel des gais et des lesbiennes.

Les recherches actuelles sur la santé mentale des homosexuel-les sont héritières de ces perspectives divergentes. D'une certaine manière, nous sommes passés d'une vision majoritaire de l'homosexualité comme trouble psychiatrique à la santé mentale des homosexuel-les comme question de santé publique. Néanmoins cette thématique de recherche reste peu développée, en particulier en France. Depuis ces dernières années, des revendications émergent pour une meilleure connaissance et prise en compte de l'ensemble des questions de santé qui concernent les gais et les lesbiennes comme en attestent, par exemple, les éditoriaux de deux numéros spéciaux sur ce thème de l'American Journal of Public Health en 2001 et 2008 [13,14].

Comprendre l'évolution sociale et les transformations des paradigmes scientifiques concernant l'homosexualité nous permet de situer historiquement les travaux de ces dix dernières années, qui sont l'objet de la présente revue. Antérieurement, peu d'enquêtes en population générale (absence de questions sur les comportements et l'orientation sexuels) ou en milieu 
homosexuel (absence de groupe de comparaison) permettaient de mettre en perspective les liens entre orientation sexuelle et santé mentale, d'où le choix d'une période récente pendant laquelle ces recherches, méthodologiquement plus élaborées, se sont développées.

L'objectif de cet article est de présenter les résultats et les éventuelles hypothèses explicatives sur les liens entre l'orientation sexuelle et la santé mentale. La notion d'orientation sexuelle, telle que nous l'entendons ici, concerne l'ensemble des individus. Elle différencie les hétérosexuel-les, orientation sexuelle majoritaire constituée en norme sociale, et les homo ou bisexuel-les, orientation minoritaire tant par le nombre que par la position sociale dévalorisée. L'orientation sexuelle est appréhendée par la manière dont les individus se définissent, au-delà du sexe de leurs partenaires. Dans cet article, les termes d'homo, bi ou hétérosexuel-les sont utilisés comme des modalités spécifiques de l'orientation sexuelle.

Des revues de la littérature sur la santé des gais et des lesbiennes ont été publiées au début des années 2000 : elles sont soit très générales, englobant l'ensemble des questions de santé et donc peu développées sur la santé mentale [15-17], soit centrées sur le seul risque suicidaire [18-20]. D'autres ne cherchent pas un bilan systématique de la littérature, tout en mettant en perspective de nombreuses enquêtes quantitatives et études cliniques [21, 22]. Il existe deux revues, parues respectivement en 2005 et 2008, l'une en français analysant des articles de 1993 à 2004 [23], et l'autre en anglais sur des articles de 1996 à 2005 [24].

La présente revue inclut des enquêtes plus récentes, plus souvent européennes. Elle vise à analyser les problèmes soulevés par la variabilité des indicateurs d'orientation sexuelle utilisés dans les enquêtes, à comparer les résultats sur la santé mentale en fonction de ces indicateurs et des échelles de mesure de santé mentale et à considérer ensemble les études sur la santé mentale et celles sur le risque suicidaire.

\section{Méthode de la revue}


Ont été sélectionnés dans Pubmed des articles publiés en langue anglaise ou française, de 1997 à 2008, à l'aide des mots-clés suivants : «homosexuality / sexual orientation et suicide, depression, mental health ». Pour être retenus, les articles devaient présenter des résultats d'enquêtes quantitatives, comparant la situation de personnes adultes « homo-bisexuelles » à celle de personnes «hétérosexuelles », le mode de définition des différents groupes étant un élément de l'analyse abordé ci-dessous.

Tous les articles présentant des données sur des femmes et sur les deux sexes ont été retenus. Ceux centrés sur les seuls hommes, souvent en lien avec la problématique de l'infection à VIH, n'ont pas été inclus. Uniquement quatre articles ne concernant que les hommes répondaient à nos critères de sélection (deux sur le risque suicidaire [25, 26], deux sur la santé mentale en général $[27,28])$. Ce choix de ne pas les inclure est dicté par la volonté de mettre en lumière les analyses sur les femmes, moins nombreuses et peu valorisées. Les nombreux articles sur des populations de "jeunes", scolarisés ou en rupture sociale, n'ont pas été retenus ici car ils nécessiteraient une revue à eux seuls. Certains résultats, essentiellement sur le risque suicidaire, sont disponibles en français [29, 30]. De plus, la sexualité et l'orientation sexuelle, qui se construisent à ces âges, sont encore labiles et, pour de nombreux jeunes, une source d'incertitude et de questionnement, qui demanderaient une analyse spécifique.

$\mathrm{Au}$ total, cette revue analyse 22 articles [31-52], dont deux portent sur la même base de données. L'information tirée de ces articles est décrite dans trois tableaux : le tableau 1 présente le descriptif des enquêtes et des échantillons, le tableau 2 les indicateurs d'orientation ou de comportements sexuels et leur distribution et le tableau 3 les indicateurs de santé, les facteurs de contexte de l'analyse et les principaux résultats.

\section{Résultats}




\subsection{Quel type d'enquêtes? (Tableau 1)}

Les données, collectées après le milieu des années 1990, proviennent essentiellement de pays anglo-saxons. Sur les 21 enquêtes incluses dans cette revue, 12 ont été réalisées aux EtatsUnis, deux en Australie, deux en Grande-Bretagne, deux en France, une aux Pays-Bas, en Nouvelle-Zélande, et au Canada (Québec).

Les travaux peuvent être regroupés en deux grandes catégories : d'une part, des analyses secondaires de données collectées dans le cadre d'enquêtes, souvent nationales, portant en général sur la santé, avec des échantillons aléatoires et de taille importante (14 enquêtes). Les informations sont, dans ce cas, le plus souvent recueillies en face à face ou par téléphone. D'autre part, des enquêtes en milieu homosexuel avec un groupe de comparaison d'hétérosexuel-les, sur des échantillons plus petits et utilisant des auto-questionnaires.

La majorité des enquêtes (treize) inclut des hommes et des femmes, qu'il s'agisse d'enquêtes en population générale ou en milieu homosexuel.

Les enquêtes concernent la population adulte, avec des bornes d'âge variables : de 15-25 ans à 59-75 ans ou plus. Quatre cohortes ont retenu des âges ou des tranches d'âge spécifiques. La Women's Health Initiative [34] porte sur des femmes âgées de 50 à 79 ans.

\subsection{Comment est définie "l’homosexualité»? Quelle est sa fréquence dans la} population étudiée? (Tableau 2)

Les indicateurs utilisés dans les différentes enquêtes pour définir l'homo-, la bi- et l'hétérosexualité sont variables. Néanmoins, ils sont tous construits à partir d'un ou plusieurs des trois indicateurs suivants : l'attirance pour l'un ou l'autre sexe, les comportements sexuels 
et, plus précisément, le sexe des partenaires et l'autodéfinition, même si les dimensions temporelles envisagées et les regroupements effectués sont divers.

La moitié des enquêtes s'appuient sur l'autodéfinition comme indicateur d'orientation sexuelle : se dire homosexuel-le, gay ou lesbienne, bisexuel-le ou hétérosexuel-le est le critère de distinction des groupes à comparer. C'est le cas pour la majorité des enquêtes réalisées en milieu homosexuel (cinq sur sept) et près de la moitié de celles conduites en population générale (six sur quatorze).

Les autres enquêtes considèrent uniquement le sexe des partenaires pour définir l'indicateur que ce soit pendant l'année précédente [32,33,37,51], lors des 5 dernières années [35] ou dans la vie entière $[34,47]$.

Une seule enquête [43] utilise uniquement l'attirance pour l'un ou l'autre sexe. Cette attirance, mesurée sur une échelle à 6 positions, proche de celle proposée par Kinsey et al. [7,8], prend en compte l'attirance actuelle et passée. Deux études conjuguent attirance et comportements sexuels $[31,39]$ et une troisième, comportements et autodéfinition [52].

Plusieurs travaux ont montré que ces indicateurs sont congruents mais qu'ils ne se recoupent pas entièrement [53,54]. Quoiqu'il en soit, les analyses menées comparent en général deux, parfois trois groupes et les découpages plus fins en termes de périodes, d'exclusivité ou non, sont regroupés principalement pour des raisons d'effectifs.

En termes de fréquence de l'homo-bisexualité, seules les enquêtes sur des échantillons représentatifs sont utilisables. Selon les études, les homosexuels et les bisexuels sont regroupés ou considérés séparément. Quand le comportement sexuel des 12 derniers mois est pris en compte les pourcentages de personnes homo-bisexuelles oscillent entre $1 \%$ et $2 \%$ pour les femmes et autour de $3 \%$ pour les hommes. Quand on considère l'autodéfinition des répondants, les fréquences d'homo-bisexualité sont de 1 à $4 \%$ pour les femmes et autour de $3 \%$ pour les hommes. 
Seule l'enquête australienne [38] fait état d'un pourcentage plus élevé de déclaration d'homosexualité, basée sur l'autodéfinition, chez les femmes que chez les hommes; ce résultat se retrouve aussi dans l'enquête sur les comportements sexuels en Australie [55]. En revanche il n'est observé dans aucun autre pays [10].

Le choix de l'indicateur ne semble pas influer de manière déterminante sur les taux de déclaration d'homo- ou bisexualité, bien que ces taux soient légèrement plus élevés quand l'autodéfinition est utilisée plutôt que le sexe des partenaires.

3.3. Quels sont les principaux indicateurs de santé mentale pris en compte? Quels sont les outils de mesure? (Tableau 3)

La consommation excessive et surtout la dépendance à l'alcool comme aux drogues illicites font partie des indicateurs classiques de santé mentale. Quand elles sont étudiées, ce qui est le cas pour 15 articles, les auteurs ne décrivent pas toujours les instruments utilisés pour les mesurer. Il s'agit majoritairement de questions ad hoc. L'Alcohol Use Disorders Identification Test (AUDIT) est utilisé dans une des études [38] et le test DETA-CAGE, en 4 questions, dans deux autres $[48,51]$. En ce qui concerne l'usage de ou la dépendance aux drogues, aucun outil standardisé de mesure n'est employé.

La plupart des études analysent la fréquence des symptômes dépressifs ou anxieux, des phobies ou attaques de panique. Le plus souvent les auteurs recourent à des instruments validés; certains sont des échelles de dépistage tels que:

- le General Health Questionnaire (GHQ) $[42,47,48]$ en 12 ou 28 items portant sur l'anxiété, la dépression ou l'asociabilité du moment ; 
- $\quad$ le Short Form (SF) 36 [34,44,46] qui est une mesure de santé générale et de capacité à accomplir les gestes de la vie quotidienne, en 36 items ; cet instrument produit 8 échelles dont quatre concernent la santé mentale actuelle ;

- le Psychiatric Symptom Index (PSI) [32] en 14 items, qui permet de mesurer les symptômes de dépression, d'anxiété, d'irritabilité et les problèmes cognitifs au cours de la dernière semaine ;

- $\quad$ le Kessler Psychological Distress Scale (K10) [52] qui mesure en dix items l'état de détresse des quatre dernières semaines, avec des questions sur les symptômes d'anxiété et de dépression.

- $\quad$ le Center for Epidemiologic Studies Depression Scale (CES-D) [34,46] qui est une mesure de dépression en 20 items qui met l'accent sur l'humeur de la dernière semaine ;

- le Brief Symptom Inventory (BSI) [36,45] en 53 items regroupés en 9 dimensions, dont la dépression, l'anxiété, la phobie, l'hostilité..., symptômes ressentis pendant la dernière semaine ;

- $\quad$ les échelles de Goldberg [38] visant à dépister la dépression et l'anxiété actuelle, en 9 items chacune, pour une population consultant en médecine générale ;

Des échelles de qualité de vie ou d'estime de soi, telle que l'échelle de Rosenberg en 10 items, sont utilisées dans certaines enquêtes [36,45].

D'autres sont des instruments issus de la clinique psychiatrique comme:

- le Composite International Diagnostic Interview-Short Form (CIDI-SF) $[33,35,37,40,49,51]$ qui interroge sur différents aspects des troubles mentaux (dépression, anxiété, pensées suicidaires) des 12 derniers mois ;

- $\quad$ le Clinical Interview Schedule (CIS) [42] qui interroge sur la présence et la sévérité de 14 symptômes non psychotiques pendant la semaine précédente : troubles 
dépressifs ou anxieux, fatigue, perte de mémoire ou troubles de la concentration, troubles du sommeil, attaques de panique, comportements compulsifs et pensées obsessionnelles.

Le recours aux soins spécialisés en santé mentale est parfois étudié, en interrogeant les sujets sur les éventuelles psychothérapies qu'ils suivent (10 articles), sur les hospitalisations en psychiatrie (un article) ou sur leur consommation de médicaments psychotropes ( 5 articles).

Les pensées suicidaires et les tentatives de suicide sont un objet d'analyse pour douze articles. Pour les tentatives de suicide, les périodes de référence sont variables: 12 derniers mois [32,35,38,46,47], vie entière $[31,35,39,43,47,49-51]$ ou définie par rapport à un âge : avant ou après 18 ans [45]. En outre King et al. [42] étudient les pensées et comportements autodestructeurs actuels.

\subsection{Concordance et discordance des principaux résultats sur la santé mentale}

\section{(Tableau 3)}

La quasi-totalité des études qui portent sur les deux sexes présentent leurs résultats séparément pour les hommes et pour les femmes. La question des facteurs à prendre en compte dans l'analyse est importante car plusieurs éléments diffèrent entre les personnes homosexuelles et les hétérosexuelles : les premières ont, en moyenne, un niveau d'études plus élevé, elles vivent plus rarement en couple, ont moins souvent des enfants et résident plus souvent dans les grandes agglomérations. Dans les articles revus ici, les stratégies d'ajustement varient : deux articles n'ont considéré aucun facteur [31,32] et un autre a adopté une stratégie d'échantillonnage - paires de sœurs- visant à contrôler les principales différences [36]. L'âge est très souvent pris en compte et dans une moindre mesure le niveau d'études. Plus rarement d'autres facteurs sont considérés comme l'origine ethnique, les 
événements de vie ou les violences subies. Les résultats commentés ci-dessous tiennent compte des ajustements (tableau 3).

\subsubsection{Alcool et drogues}

La majorité des enquêtes, sur échantillon représentatif ou non, montre une consommation ou un abus d'alcool et un usage de drogues plus fréquents chez les femmes homosexuelles ou bisexuelles que chez les hétérosexuelles. Ce résultat n'est généralement pas retrouvé pour les hommes en ce qui concerne l'alcool, mais l'est pour les drogues. Seuls quatre articles, utilisant des outils de mesure différents, standardisés comme l'AUDIT et le DETA-CAGE ou basés sur des questions ad hoc, ne décrivent aucune différence dans les consommations ou dépendance à l'alcool selon l'orientation sexuelle, pour les deux sexes [38,40,50,51].

\subsubsection{Dépression et anxiété}

Vingt articles présentent des résultats sur la dépression, l'anxiété ou la détresse psychologique, le stress et la santé mentale perçue. Ces résultats sont très contrastés.

Six articles très majoritairement sur des échantillons de femmes, n'observent pas de différence selon l'orientation sexuelle, à l'âge adulte ou dans une période récente, pour les symptômes dépressifs $[31,36,41,45,47,50]$.

Les autres articles montrent des différences. Parmi les études ne portant que sur les femmes, trois articles décrivent une moins bonne santé mentale, tant pour la dépression que pour l'anxiété, chez les non-hétérosexuelles [34,44,46]; certains insistent sur les plus mauvais scores des femmes bisexuelles pour la dépression et l'anxiété [44]. Parmi les enquêtes incluant des hommes et des femmes, quatre signalent une plus mauvaise santé mentale des homosexuels, femmes comme hommes [37,38,42,52]. Cinq rapportent globalement des différences significatives pour les hommes mais pas pour les femmes, avec quelques 
variations selon les symptômes considérés [32,33,40,43,51] et une seule observe des différences, sur la dépression et les troubles anxieux, pour les femmes et pas pour les hommes [35].

Les études qui ne trouvent pas de différence de dépression ou d'anxiété selon l'orientation sexuelle sont majoritairement des études qui recrutent les participants homosexuel-les dans des structures communautaires. Les enquêtes sur échantillon représentatif trouvent toutes une différence, sauf deux [41,47], elles sont plus directement centrées sur la dépression.

\subsubsection{Consommation de psychotropes et psychothérapies}

La grande majorité des douze articles qui prennent en compte le recours aux soins de santé mentale ou l'usage de psychotropes décrivent un recours plus fréquent pour les homobisexuel-les, femmes comme hommes. Deux articles [41,47] n'observent pas de différence entre les homo-bisexuelles et les hétérosexuelles quand au recours à la psychothérapie et un autre signale que les lesbiennes ont eu un recours plus fréquent aux soins de santé mentale dans le passé mais pas actuellement [31].

\subsubsection{Tentatives de suicide}

Sur les douze articles étudiant les tentatives de suicide, le plus grand nombre observe que celles-ci sont plus fréquentes chez les femmes et les hommes homo-bisexuel-le-s que chez les hétérosexuel-le-s [31,32,38,39,45-47,50]. Quatre articles montrent que les tentatives de suicide sont plus fréquentes chez les hommes homosexuels mais pas chez les femmes $[35,43,49,51]$.

\section{Discussion}


Cette revue de la littérature fait le bilan des études, publiées très majoritairement en langue anglaise dans ces 15 dernières années, sur l'état de santé mentale en relation avec l'orientation sexuelle. Pour figurer dans cette revue, les études devaient répondre à certaines exigences méthodologiques, en particulier disposer d'un groupe de référence - personnes hétérosexuelles - recruté dans les mêmes conditions que le groupe étudié - personnes homosexuelles - et pour lequel les auteurs disposaient des mêmes informations relatives à la santé mentale. La présence de deux types d'enquêtes dans cette revue permet d'une part d'estimer avec une certaine précision les fréquences des problèmes de santé mentale dans les deux groupes (enquêtes en population générale) et ainsi de réduire le risque de biais des enquêtes ciblées, signalé dans d'autres revues de la littérature [15,17,21,22]. D'autre part les enquêtes sur échantillon "communautaire" apportent des informations plus précises sur une population souvent mal appréhendée par les enquêtes en population générale [56].

Les articles analysés font tous état de possibles biais liés à la variabilité des définitions de l'orientation sexuelle. C'est dans les années 2000 que la question de l'auto-identification devient très majoritairement le critère utilisé pour définir les groupes. Ceci est surtout valable dans le monde anglo-saxon, les enquêtes en France et en Hollande utilisant seulement le critère du sexe du partenaire. Il faudra attendre l'enquête Contexte de la Sexualité en France, non incluse dans cette revue [57] pour que la question de l'auto-identification soit posée dans une enquête en population générale en France.

A l'exception de deux enquêtes [31,39] qui utilisent une combinaison de l'attirance et du comportement, toutes les enquêtes sur des échantillons ciblés prennent l'autodéfinition comme mesure de l'orientation sexuelle dès les années 1990. On peut supposer que l'introduction de questions sur l'auto-définition dans les enquêtes en population générale s'est faite à partir de ces expériences plus «communautaires » de définir l'homosexualité à partir de ce que les personnes concernées disent d'elles-mêmes. De plus, s'identifier comme gai ou 
lesbienne marque le fait d'accepter, voire de revendiquer une désignation stigmatisante et de faire partie d'un groupe, que l'on fréquente ou non les lieux de sociabilité homosexuelle.

Quoi qu'il en soit, malgré le manque d'homogénéité des indicateurs utilisés pour définir l'orientation sexuelle, il existe un consensus minimum sur les trois principales dimensions de l'orientation sexuelle : attirance, comportements et auto identification [53,54]. Le "choix" de telle ou telle dimension à inclure dans le questionnaire, pour les enquêtes en population générale, est souvent moins une décision théorique argumentée qu'un compromis sur la taille du questionnaire, la pertinence au vu du thème étudié, les présupposés sur l'acceptation de certaines questions par les interviewés, voire les financeurs. Quand l'ensemble des indicateurs est disponible dans le questionnaire, l'utilisation de l'un ou de l'autre pour la publication des résultats est souvent fonction des effectifs de personnes concernées. Quand la taille de l'échantillon n'est pas très importante, les homo et bisexuel-les sont souvent regroupés, qu'ils soient par ailleurs construits comme groupe par leur comportement sexuel ou par leur autodéfinition. L'ensemble de ces contraintes, techniques, intellectuelles et politiques, rend difficile la mise en place d'un réel consensus sur cette question. Il semble néanmoins qu'à minimum, deux mesures distinctes de l'orientation sexuelle soient nécessaires, l'une comportementale et l'autre identitaire, afin de mieux évaluer l'impact, éventuellement différent, de chacune sur la santé [58-60].

Les résultats sur les relations entre l'orientation sexuelle et les troubles de la santé mentale sont contrastés. De manière générale, ils n'apparaissent pas différents selon le type d'instrument utilisé tant pour évaluer la santé mentale que pour définir les groupes selon l'orientation sexuelle, ni selon les pays. Par contre le type d'échantillon semble influer sur les résultats, les enquêtes en milieu homosexuel avec groupe de contrôle rapportent peu de différences entre les homo-bisexuel-les et les autres. On peut penser que ces enquêtes 
recrutent des personnes plus affirmées et plus intégrées dans des réseaux de sociabilité homosexuels, sélectionnant ainsi des personnes en meilleure santé psychique.

Il faut souligner le fait que, quand une différence est trouvée, en général dans les enquêtes en population générale, les résultats sont plus nets - épisode dépressif ou détresse psychologique plus fréquents pour les non-hétérosexuels - pour les hommes que pour les femmes.

Les résultats sont beaucoup plus concordants en ce qui concerne les tentatives de suicide dont le risque est plus élevé pour les hommes et les femmes homosexuels. Toutes les études montrent que cet excès de risque est significatif pour les hommes, et trois quart d'entre elles pour les femmes. On peut penser que comme les femmes en général sont touchées plus fréquemment que les hommes par le risque suicidaire (pensées suicidaires et tentatives de suicide) les différences selon l'orientation sexuelle seraient moins visibles pour elles. Par contre il faut signaler que la mortalité par suicide est plus élevée chez les hommes [61], et la rareté des études sur les suicides accomplis selon l'orientation sexuelle ne permet pas de conclusion définitive sur ce plan.

Les auteurs n'expliquent que partiellement ces différences de santé mentale. Certains insistent sur le fait que la grande majorité des homos-bisexuel-les n'ont pas de problèmes particuliers de santé mentale [33], rompant ainsi avec la vieille tradition psychiatrisante. Un seul article évoque de possibles causes biologiques et le niveau atypique des androgènes prénataux selon le sexe [37]. Pour tenter de comprendre le risque accru néanmoins constaté, les mêmes hypothèses sont avancées : discrimination, stigmatisation, absence de soutien social. Il est probable que l'hostilité sociale que la plupart des homosexuels expérimentent est, au moins en partie, une raison de leurs taux plus élevés de souffrance psychologique. Plusieurs auteurs évoquent la notion de « stress minoritaire» proposée par Meyer [62]. Certains groupes minoritaires doivent faire face à des facteurs de stress chronique résultant de 
leur statut défavorisé dans la société [63]. Les homosexuel-les peuvent en plus être confrontés à un rejet de leur famille, rejet qui ne frappe pas d'autres groupes minoritaires, comme par exemple ceux fondés sur la couleur de peau, qui, en général, partagent cette position avec les membres de leur famille.

Les personnes homo-bisexuelles, moins inscrites dans les institutions sociales de l'hétérosexualité, ont souvent des modes de vie différents des hétérosexuels -partenaires sexuels plus nombreux $[32,47,48,64]$ et relations de couple plus courtes ou plus instables $[36,38,42,50]$, avec les ruptures et les difficultés que cela implique, usage d'alcool ou de drogues plus courant $[32-34,37,42-44,47,48]$. Il est possible que ces modes de vie, plus marginaux, les exposent à une détresse psychologique. De plus, certaines études ont montré que le risque d'avoir subi des violences ou du harcèlement était plus fréquent chez les personnes non hétérosexuelles, en particulier pour les femmes [21,31,39,42,47,65].

Enfin on doit évoquer un aspect sociologique et méthodologique en supposant que certains répondants auraient une plus grande capacité que d'autres à « tout dire », à déclarer des faits généralement tus; ainsi ces répondants déclareraient à la fois des comportements homosexuels et des tentatives de suicide ou des symptômes dépressifs [43].

Il est difficile d'évoquer la situation des personnes bisexuelles, car elles sont généralement peu nombreuses et incluses dans le même groupe que les homosexuel-le-s. Toutefois, 11 articles les distinguent dans leur présentation des résultats [32,34,37,38,41,44, 45,46,48,50,52]; parmi eux, 8 montrent que la santé mentale, que ce soit en termes de détresse psychologique, d'anxiété ou de tentatives de suicide, est moins bonne pour les personnes bisexuelles que pour les homosexuel-le-s, résultat retrouvé pour les hommes et pour les femmes. Certains auteurs soulignent que le fait d'avoir une orientation ni hétérosexuelle ni clairement homosexuelle constitue une source importante de stress, en plus des pressions sociales liées à une orientation sexuelle minoritaire [38]. Les personnes se définissant comme 
bisexuelles feraient l'objet d'une double discrimination aussi bien dans les communautés homosexuelles que dans la société en général [36,58]. Ainsi il est possible que ces personnes taisent davantage leur orientation sexuelle ; or les recherches ont montré une relation entre ne pas faire connaître ses choix sexuels et une moins bonne santé mentale [50].

En conclusion cette revue montre un tableau assez cohérent d'une moins bonne santé mentale pour les personnes homo-bisexuelles, les études sont particulièrement convergentes à propos du risque plus élevé de tentatives de suicide. Néanmoins, comme le soulignent plusieurs auteurs, la tendance à déduire du taux plus élevé de détresse psychologique chez les homobisexuel-les que l'homosexualité elle-même serait une maladie mentale n'a pas entièrement disparue [22,62]. Les travaux ultérieurs devraient étudier les facteurs de risque ainsi que de protection des troubles de la santé mentale dans la population homo-bisexuelle : expériences de discrimination, rôle du soutien social, du coming out, etc. La manière dont ces risques varient selon le sexe, le genre (mise en question ou non des rôles attribués à chaque sexe, conformité ou non à la définition et présentation de soi, socialement prescrite, censée être différente pour les hommes et pour les femmes [66]), ainsi que selon l'âge (en termes d'évolution personnelle et aussi de moment historique), l'origine ethnique ou les conditions socio-économiques est un élément essentiel pour la compréhension du lien entre orientation sexuelle et santé mentale. Il est important que les grandes enquêtes en population sur la santé incluent des mesures de l'orientation sexuelle, en particulier en France où ce type de données est pour le moins lacunaire. Données qui permettraient d'étudier de manière plus détaillée les questions de santé dans divers groupes et dans différents milieux de vie, et ainsi de mettre en place des politiques de santé publique adaptées. 


\section{Références}

[1] Boehmer U. Twenty years of public health research: inclusion of lesbian, gay, bisexual and transgender populations. Am J Public Health 2002; 92:1125-30.

[2] Krafft-Ebing R von. Psychopathia Sexualis. Paris: Payot, 1969 (1 $1^{\text {ère }}$ édition 1887).

[3] Hirschfeld M. Les homosexuels de Berlin. Lille: Cahiers Gai-Kitsch-Camp, 1990 (1 ${ }^{\text {ère }}$ édition 1908).

[4] Hooker E. Reflections of a 40-year exploration. A scientific view on homosexuality. Am Psychol 1993;48 :450-3.

[5] Saghir MT, Robins E, Walbran B, Gentry KA. Homosexuality IV. Psychiatric disorders and disability in the female homosexual. Am J Psychiatry 1970; 127:147-54.

[6] Duberman M. Cures. A gay man Odyssey. New York: Penguin Books, 1991.

[7] Kinsey AC, Pomeroy WB, Martin CE. Sexual behavior in the human male. Philadelphia: Saunders, 1948.

[8] Kinsey AC, Pomeroy WB, Martin CE, Gebhard PH. Sexual behavior in the human female. Philadelphia: Saunders, 1953.

[9] Gagnon JH. Gender preference in erotic relations: the Kinsey scale and sexual scripts. In: McWhirter DP, Sanders SA, Reinisch JM, eds. Homosexuality/heterosexuality: concepts of sexual orientation. New York: Oxford University Press, 1990: 177-207.

[10] Michael S, Lhomond B. Conceptualization and measurement of homosexuality in sex surveys : a critical review. Cad Saude Publica 2006;22:1365-74.

[11] Lauritsen J, Thorstad D. The early homosexual rights movement (1864-1935). New York: Times Change Press, 1974.

[12] FHAR. Rapport contre la normalité. Paris: Editions Champ Libre, 1971.

[13] Meyer IH. Why lesbian, gay, bisexual, and transgender public health? Am J Public Health 2001;91:856-9. 
[14] Auerbach J. Lesbian, gay, bisexual, and transgender public health: progress and change. Am J Public Health 2008;98:970.

[15] Dean L, Meyer IH, Robinson K, Sell RL, Sember R, Silenzio VMB et al. Lesbian, gay, bisexual and transgender health: findings and concerns. J Gay Lesbian Med Assoc 2000;4:101-51.

[16] Bernhard LA. Lesbian health and health care. Annu Rev Nurs Res 2001;19:145-77.

[17] Roberts SJ. Lesbian health research: a review and recommendations for future research. Health Care Women Int 2001;22:537-52.

[18] Saunders JM, Valente SM. Suicide risk among gay men and lesbians: a review. Death Stud 1987;11:1-23.

[19] McDaniel JS, Purcell D, D’Augelli A. The relationship between sexual orientation and risk for suicide: research findings and future directions for research and prevention. Suicide Life-Threat Behav 2001;31(supp): 84-105.

[20] McAndrew S, Warne T. Ignoring the evidence dictating the practice: sexual orientation, suicidality and the dichotomy of the mental health nurse. J Psychiatr Ment Health Nurs 2004;11:428-34.

[21] Cochran SD. Emerging issues in research on lesbians and gay men's health: does sexual orientation matter? Am Psychol 2001;56:931-47.

[22] Herek GM, Garnets LD. (2007) Sexual orientation and mental health. Annu Rev Clin Psychol 2007;3:353-75.

[23] Julien D, Chartrand E. Recension des études utilisant un échantillon probabiliste sur la santé des personnes gaies, lesbiennes et bisexuelles. Can Psychol 2005;46:235-50.

[24] King M, Semlyen J, Tai SS, Killaspy H, Osborn D, Popelyuk D, Nazareth I. A systematic review of mental disorder, suicide, and deliberate self harm in lesbian, gay and bisexual people. BMC Psychiatry 2008;8:70. 
[25] Bagley C, Tremblay P: Suicidal behaviors in homosexual and bisexual males. Crisis 1997, 18(1):24-34.

[26] Herrell R, Goldberg J, True WR, Ramakrishnan V, Lyons M, Eisen S, Tsuang MT:

Sexual orientation and suicidality: a co-twin control study in adult men. Arch Gen Psychiatry 1999,56(10):867-74.

[27]Cochran SD, Mays VM: Lifetime prevalence of suicide symptoms and affective disorders among men reporting same-sex sexual partners: Results from NHANES III. Am J Public Health 2000, 90(4):573-8.

[28] Russell CJ, Keel PK. Homosexuality as a specific risk factor for eating disorders in men. Int J Eat Disord 2002,31:300-6

[29] Lhomond B, Michaels S, Levinson S, Mailloux M. Jeunes et sexualités. Rapport pour la Direction Générale de la Santé et le Ministère de la Jeunesse et des Sports, multigraphié 2003.

[30] Verdier E, Firdion JM. Homosexualités et suicide. Etudes, témoignages et analyse. Paris: H\&O Editions, 2003.

[31] Hughes TL, Pollinger Haas A, Avery L. Lesbians and mental health: preliminary results from the Chicago women's health survey. J Gay Lesbian Med Assoc 1997;1:137-48.

[32] Clermont M, Lacouture Y. Orientation sexuelle et santé. In: Institut de la Statistique du Québec, Enquête sociale et de santé 1998. Québec: Gouvernement du Québec 2000: 21930.

[33] Cochran SD, Mays VM. Relation between psychiatric syndromes and behaviorally defined sexual orientation in a sample of the US population. Am $\mathbf{J}$ Epidemiol 2000;151:516-23. 
[34] Valanis BG, Bowen DJ, Bassford T, Whitlock E, Charney P, Carter RA. Sexual orientation and health: comparisons in the Women's Health Initiative Sample. Arch Fam Med 2000;9:843-53

[35] Gilman SE, Cochran SD, Mays VM, Hughes M, Ostrow D, Kessler RC. Risk of psychiatric disorders among individuals reporting same-sex sexual partners in the National Comorbidity Survey. Am J Public Health 2001;91:933-9.

[36] Rothblum ED, Factor R. Lesbians and their sisters as a control group: demographic and mental health factors. Psychol Science 2001;12:63-9.

[37] Sandfort TGM, Graaf R de, Bijl RV, Schnabel P. Same-sex sexual behavior and psychiatric disorders. Findings from the Netherlands Mental Health Survey and incidence study (NEMESIS). Arch Gen Psychiatry 2001;58:85-91

[38] Jorm AF, Korten AE, Rodgers B, Jacomb PA, Christensen H. Sexual orientation and mental health: results from a community survey of young and middle-aged adults. $\mathrm{Br} \mathbf{J}$ Psychiatry 2002;180:423-7.

[39] Matthews AK, Hughes TL, Johnson T, Razzano LA, Cassidy R. Prediction of depressive distress in a community sample of women: the role of sexual orientation. Am J Public Health 2002;92:1131-9.

[40] Cochran SD, Sullivan JG, Mays VM. Prevalence of mental disorders, psychological distress, and mental health services use among lesbian, gay, and bisexual adults in the United States. J Consult Clin Psychol 2003;71:53-61.

[41] Diamant AL, Wold C. Sexual orientation and variation in physical and mental health status among women. J Womens Health 2003;12:41-9.

[42] King M, McKeown E, Warner J, Ramsay A, Johnson K, Cort C, et al. Mental health and quality of life of gay men and lesbians in England and Wales. Br J Psychiatry 2003; 183: $552-8$ 
[43] Skegg K, Nada-Raja S, Dickson N, Paul C, Williams S. Sexual orientation and self-harm in men and women. Am J Psychiatry 2003; 160: 541-6.

[44] Case P, Austin B, Hunter DJ, Manson JE, Malspeis S, Willett WC et al. Sexual orientation, health risk factors, and physical functioning in the Nurses Health Study II. J Womens Health 2004;13:1033-47.

[45] Balsam KF, Beauchaine TP, Mickey RM, Rothblum ED. Mental health of lesbian, gay, bisexual and heterosexual siblings: effects of gender, sexual orientation and family. $\mathbf{J}$ Abnorm Psychol 2005;114:471-6.

[46] McNair R, Cavanagh A, Agius P, Tong B. The mental health status of young adult and mid-life non-heterosexual Australian women. Aust N Z J Public Health 2005;29:265-71.

[47] Lhomond B, Saurel-Cubizolles MJ. Violence against women and suicide risk: the neglected impact of same-sex sexual behaviour. Soc Sci Med 2006;62:2002-13.

[48] King M, Nazareth I. The health of people classified as lesbian, gay and bisexual attending family practitioners in London: a controlled study. BMC Public Health 2006;6:127.

[49] de Graaf R, Sandfort TGM, ten Have M. Suicidality and sexual orientation: differences between men and women in general population-based sample from the Netherlands. Arch Sex Behav 2006;35:253-62.

[50] Koh AS, Ross LK. Mental health issues: a comparison of lesbian, bisexual and heterosexual women. J Homosex 2006;51:33-57.

[51] Jouvin E, Beaulieu-Prévost D, Julien D. Minorités sexuelles : des populations plus exposées que les autres ? In INPES. Baromètre Santé 2005.Paris:INPES; 2007:271-84.

[52] Cochran SD, Mays VM. Physical health complaints among lesbians, gay men, and bisexual and homosexually experienced heterosexual individuals: results from the California Quality of Life Survey. Am J Public Health 2006;97:2048-55. 
[53] Laumann EO, Gagnon JH, Michael RT, Michaels S. The social organization of sexuality: sexual practices in the United States. Chicago: University of Chicago Press, 1994.

[54] Brogan D, Frank E, Elon L, O’Hanlan KA. Methodological concerns in defining lesbian for health research. Epidemiology 2001;12:109-13.

[55] Smith AM, Rissel CE, Richters J, Grulich AE, de Visser RO. Sex in Australia: sexual identity, sexual attraction and sexual experience among a representative sample of adults. Aust N Z J Public Health 2003;27:138-45.

[56] Rothblum ED, "I only read about myself on bathroom walls":the need for research on mental health of lesbians and gay men. J Consult Clin Psychol 1994;62:213-20.

[57] Bajos N, Beltzer N. Les sexualités homo-bisexuelles : D'une acceptation de principe aux vulnérabilités sociales et préventives. In Bajos N, Bozon M, eds. Enquête sur la sexualité en France .Pratiques, genre et santé. Paris: La Découverte, 2008:243-95.

[58] Morris JF, Rothblum ED. Who fills out a "lesbian" questionnaire ? The interrelationship of sexual orientation, years out, disclosure of sexual orientation, sexual experience with women, and participation in the lesbian community. Psychol Women Q 1999;33:537-57.

[59] Midanik LT, Drabble L, Trocki K, Sell RL. Sexual orientation and alcohol use:identity versus behavior mesures. J LGBT Health Res 2007;3:25-35.

[60] Mayer KH, Bradford JB, Makadon HJ, Stall R, Goldhammer H, Landers S. Sexual and gender minority health: what we know and what needs to be done. Am J Public Health 2008;98:989-95.

[61] Cousteaux AS, Pan Ke Shon JL. Le mal-être a-t-il un genre? Suicide, risque suicidaire, dépression et dépendance alcoolique. Revue française de Sociologie 2007;45:53-92.

[62] Meyer IH. Prejudice, social stress, and mental health in lesbian, gay, and bisexual populations: conceptual issues and research evidence. Psychol Bull 2003;129: 674-97. 
[63] Mays VM, Cochran SD. Mental health correlates of perceived discrimination among lesbian, gay and bisexual adults in the United States. Am J Public Health 2001; 91:186976.

[64] Sandfort T. Homosexual and bisexual behavior in european countries. In: Hubert M, Bajos N, Sanfort T eds. Sexual behavior and HIV/AIDS in Europe. Comparisons of national surveys. London: UCL Press ;1998:68-105.

[65] SOS Homophobie. Rapport de l'enquête sur la lesbophobie. Paris, 2008, multigraphié.

[66] Erwin K. Interpreting the evidence: Competing paradigms and the emergence of lesbian and gay suicide as a « social fact ». Int J Health Serv 1993; 23:437-53. 